\title{
Gastrointestinal Bleeding Following Left Ventricular Assist Device (LVAD) Implantation: Taking the Pulse of the Problem
}

\author{
Benjamin Cassell ${ }^{1} \cdot$ Vladimir M. Kushnir $^{1}$
}

Published online: 24 July 2015

(c) Springer Science+Business Media New York 2015

Over the course of the last decade, implantation of left ventricular assist devices (LVADs) has become an increasingly popular treatment strategy for patients with advanced heart failure, both as a bridge to heart transplantation and as a "destination" therapy. The first generation of LVADs, based on a pulsatile mechanism, was plagued by poor reliability and high infection and thrombosis rates, leading to the development of smaller and more reliable continuous-flow (CF) LVADs. Following the introduction of CF-LVADs, postimplantation survival rates improved to as high as 50-75\% at 2 years [1]. Not surprisingly, CF-LVAD implantation rates have grown exponentially. With the rapidly expanding use of LVADs, it has become clear that long-term mechanical circulatory support is associated with gastrointestinal (GI) bleeding, now considered one of the more common and recalcitrant longterm complications following LVAD implantation, with a recent meta-analysis reporting the prevalence of GI bleeding near $30 \%$ [2].

While the scope of the problem is well described, the pathogenesis of GI bleeding following LVAD implantation is still uncertain. Most studies report that GI bleeding was far more prevalent in patients supported by $\mathrm{CF}$ rather than pulsatile LVADs [3]. Continuous, non-pulsatile flow of blood through the vasculature differs from the normal physiologic state, with significant pathophysiologic implications. An important concept for CF-LVADs is the pulsatility index, a measure of the variation in pulse pressure

Vladimir M. Kushnir

vkushnir@wustl.edu

1 Division of Gastroenterology, Washington University School of Medicine, 660 South Euclid Ave., Campus Box 8124, Saint Louis, MO 63110, USA representing the contribution of the native heart to the cardiac output. Interestingly, most prior studies have reported an inverse relationship between the pulsatility index and the risk of GI bleeding, patients with more pulsatile blood flow less likely to bleed [3, 4]. Although the pathophysiologic link between non-pulsatile flow and an increased propensity to bleed remains incompletely understood, recent investigation has uncovered several potential contributing factors such as the development of an acquired von Willebrand disorder in LVAD patients [5], apparently related to the continuous-flow pattern, similar to patients with aortic stenosis and Heyde's syndrome [5].

In this issue of Digestive Diseases and Sciences, Jabbar et al. [6] report a single-center, retrospective cohort study describing the incidence and outcomes of GI bleeding in patients during long-term support with CF-LVADs, representing one of the largest published experiences to date. The authors reviewed the records of 112 patients who were followed for a median of 12 months after implantation of the Heart Mate II continuous-flow LVAD. Overall, GI bleeding was reported in $39 \%$ of patients, with the first episode typically occurring within 2 months of implantation. As in most prior reports, GI bleeding was overt in a majority of cases (73\%), and the source of bleeding identified with upper endoscopy in $61 \%$ of patients. Gastro-duodenal erosive disease (erosions or ulcers) was the most frequently observed bleeding lesions, accounting for $38 \%$ of events, followed by angioectasia. Although endoscopic therapy was performed in $39 \%$ of patients, endoscopic therapy did not influence the rate of recurrent GI bleeding. Multivariable analysis identified elevated right heart pressures and post-LVAD ejection fraction as risk factors for GI bleeding.

Overall, the findings of this study are in line with comparable other recent reports. The most clinically relevant 
findings are: (1) The rate of GI bleeding is extraordinarily high following LVAD placement, approaching $40 \%$ in the current study, and (2) endoscopic therapy is ineffective in preventing recurrent bleeding in this population. Both findings appear to be a consequence of the underlying physiologic changes that accompany non-pulsatile blood flow following CF-LVAD implantation.

Notably, the authors of the accompanying article propose several new theories in order to explain GI bleeding following LVAD placement [6]. The first and most intriguing hypothesis is based on the proximity of the LVAD output to the celiac trunk. Jabbar et al. cited Poiseuille's equation $\left(Q=\Delta P \pi r^{4} / 8 L \eta\right)$ [which explains the factors including the flow of fluids in a cylindrical vessel] to argue that the decreased distance (L) from LVAD outflow to the celiac axis on the aorta (when compared to the aortic valve) increases flow $(Q)$ and pressure $(\Delta P)$ through the celiac system, combined with a capillary network accommodated to low flow from prolonged inadequate cardiac output, and increases the probability that capillary damage and angiodysplasia formation occur in response to the suddenly restored flow, explaining the preponderance of these lesions in the upper GI tract. The continuous high blood flow and large arteriovenous (AV) pressure gradient may also produce ischemic mucosal damage in the foregut, as evidenced by the high incidence of gastro-duodenal erosive disease following LVAD implantation [3].

The second hypothesis is based on the observation that patients with elevated right atrial pressures were more likely to experience GI bleeding. Jabbar et al. [6] propose that elevated right heart pressures lead to congestive hepatopathy, with consequent hepatic dysfunction and coagulopathy, increasing the risk of bleeding. Yet, this hypothesis was not supported by their observation that an international normalized ratio (INR) $>3$ was not associated with a higher incidence of GI bleeding. Furthermore, measures of hepatic function in the cohort were not available. Moreover, recent studies show that markers of hepatic function such as albumin and bilirubin improve following CF-LVAD implantation in patients with and without right heart dysfunction $[7,8]$. Taken together, these lines of evidence suggest that right ventricular dysfunction may contribute to LVAD-related GI bleeding by increasing postsinusoidal portal pressure, increasing pressures in the splanchnic circulation and potentially forming angiodysplasia throughout the GI tract, with few data supporting the role of congestive hepatopathy or coagulopathy.

A final point that bears discussion is the relationship between post-LVAD GI bleeding and anticoagulation. Similar to a previous study [2], Jabbar et al. did not find an elevated INR $(\geq 3)$ to be a significant predictor of bleeding [2]. The lack of an association between degree of coagulopathy and GI bleeding post-LVAD was best illustrated in a study by Shrode et al. [9], which reported that bleeding rates among LVAD patients were four times higher than a control group of patients receiving anticoagulation therapy following valve-replacement surgery. This lack of association further supports the central contribution of alterations of mucosal hemodynamics in post-LVAD GI bleeding.

The findings in this paper underscore that the etiology of GI bleeding post-LVAD, despite numerous studies, remains unknown [6], with the means of preventing and treating GI bleeding in this patient population remaining undetermined. Since endoscopic therapy does not prevent recurrent GI bleeding, and given the very high incidence of bleeding, an approach geared toward prevention of bleeding events is likely more appropriate. Medical therapy is appealing in this regard, but data for the two agents often used to treat angiodysplasias in non-LVAD patients, octreotide and thalidomide, are equivocal and absent, respectively [2]. Given the putative role that non-pulsatile flow has in causing bleeding, Jabbar et al. (and the authors of other papers) support increasing the pulsatility index by decreasing the pump speed of the LVAD. Although this approach has been attempted in two series as part of a multifaceted approach to management along with octreotide, endoscopic therapy and epinephrine infusion $[10,11]$, the absence of a comparator group in these studies obscured the contribution of pump speed toward hemostasis. Even in studies in which a correlation between pulsatility index and bleeding was established, pump speed was not a significant predictor of bleeding [2].

In summary, this study adds to the current body of literature on LVAD-related GI bleeding, confirming a very high incidence of bleeding and relative futility of endoscopic therapy in preventing recurrent bleeding [6]. Moreover, the proposed association between altered hemodynamics inherent in the CF-LVAD device and higher rates of GI bleeding reinforces the supposition that medical or endoscopic therapies targeted at individual lesions are unlikely to be effective. Jabbar et al. offer several intriguing hypotheses to explain LVAD-related GI bleeding, proposing possible avenues for further intervention, which will hopefully lead to prospective studies in this patient population comparing hemodynamic and hemostatic factors in patients with and without GI bleeding. In the meantime, managing patients with LVAD-related GI bleeding remains challenging, given their underlying medical complexity and the lack of evidencebased treatment options. 


\section{References}

1. Slaughter MS, Rogers JG, Milano CA, et al. Advanced heart failure treated with continuous-flow left ventricular assist device. N Engl J Med. 2009;361:2241-2251.

2. Draper KV, Huang RJ, Gerson LB. GI bleeding in patients with continuous-flow left ventricular assist devices: a systematic review and meta-analysis. Gastrointest Endosc. 2014;80:435446.

3. Kushnir VM, Sharma S, Seceombe J, et al. Evaluation of GI bleeding after implantation of left ventricular assist device. Gastrointest Endosc. 2012;75:973-979.

4. Wever-Pinzon O, Selzman CH, Drakos SG, et al. Pulsatility and the risk of nonsurgical bleeding in patients supported with the continuous-flow left ventricular assist device HeartMate II. Circ Heart Fail. 2013;6:517-526.

5. Uriel N, Pak SW, Jorde UP, et al. Acquired von Willebrand syndrome after continuous-flow mechanical device support contributes to a high prevalence of bleeding during long-term support and at the time of transplantation. J Am Coll Cardiol. 2010;56:1207-1213.

6. Jabbar HR, Abbas A, Ahmed M, et al. The incidence, predictors and outcomes of gastrointestinal bleeding in patients with left ventricular assist device (LVAD). Dig Dis Sci. (Epub ahead of print). doi:10.1007/s10620-015-3743-4.

7. Deo SV, Sharma V, Altarabsheh SE, et al. Hepatic and renal function with successful long-term support on a continuous flow left ventricular assist device. Heart Lung Circ. 2014;23:229-233.

8. Russell SD, Rogers JG, Milano CA, et al. Renal and hepatic function improve in advanced heart failure patients during continuous-flow support with the HeartMate II left ventricular assist device. Circulation. 2009;120:2352-2357.

9. Shrode CW, Draper KV, Huang RJ, et al. Significantly higher rates of gastrointestinal bleeding and thromboembolic events with left ventricular assist devices. Clin Gastroenterol Hepatol. 2014;12:1461-1467.

10. Demirozu ZT, Radovancevic R, Hochman LF, et al. Arteriovenous malformation and gastrointestinal bleeding in patients with the HeartMate II left ventricular assist device. J Heart Lung Transplant. 2011;30:849-853.

11. Hayes HM, Dembo LG, Larbalestier R, O’Driscoll G. Management options to treat gastrointestinal bleeding in patients supported on rotary left ventricular assist devices: a single-center experience. Artif Organs. 2010;34:703-706. 